\title{
100 Jahre Allergie: Clemens von Pirquet - sein Allergiebegriff und das ihm zugrunde liegende Krankheitsverständnis
}

\author{
Teil 2: Der Pirquet'sche Allergiebegriff
}

\author{
Benedikt Huber \\ Medizinische Universitäts-Kinderklinik Bern, Bern, Schweiz
}

Eingegangen am 3. April 2006, angenommen nach Revision am 9. August 2006

(C) Springer-Verlag 2006

\begin{abstract}
„Es ist nicht nur ein hoher Genuss, den Gedankengängen eines Großen zu folgen, das Bild seiner Persönlichkeit aufstrahlen zu lassen, sondern es ist eine wichtige Anregung zu eigener Kreativität, wenn man das Gewordene versteht.“ [1]

\section{Einleitung}

Nach der Darstellung von Leben und Werk Pirquets im ersten Teil dieser Arbeit [2] widmet sich der vorliegende zweite Teil vor allem dem Allergiebegriff Pirquets und dessen Entwicklung auf dem Boden seiner entscheidenden wissenschaftlichen Vorarbeiten. Dieser weicht in seiner ursprünglichen Bedeutung erheblich von dem in der heutigen Medizin verwendeten Allergiebegriff ab. Um Missverständnisse und Widersprüche zu vermeiden, wird der umfassende Allergiebegriff im Sinne Pirquets in dieser Arbeit deshalb als „Pirquet'scher Allergiebegriff“
\end{abstract} bezeichnet.

\section{Die Vorarbeiten (1901-1906) - Der Organismus im Zentrum neuer Theorien}

Überblickt man das gesamte wissenschaftliche Werk Pirquets, dann erkennt man, dass seine „Allergieforschung" nicht erst mit der Einführung des Allergiebegriffs im Jahr 1906 beginnt. Der von ihm geschaffene Begriff „Allergie“ und das damit verbundene Immunologiekonzept sind das Ergebnis einer mehrjährigen Forschungstätigkeit im Bereich der Infektiologie und Immunologie, welche von zwei Seiten her beeinflusst war: einerseits (von außen) durch die aktuellen Strömungen der medizinischen Wissenschaft am Übergang vom 19. zum 20. Jahrhundert und andererseits (von innen) durch die bereits im ersten Teil dieser Arbeit beschriebene besondere Forschungsweise Pirquets. Bedeutende Fortschritte auf dem Gebiet der Bakteriologie durch Persönlichkeiten wie Pasteur, Koch, Ehrlich und Escherich sowie wichtige immunologische Entdeckungen besonders durch die Physiologen Richet, Portier und Arthus kennzeichneten das medizinisch-wissenschaftliche Mi- lieu der damaligen Zeit [3]. Davon geprägt wendete sich Pirquet zunächst der Erforschung der Infektionskrankheiten zu und entschied sich damit für die damals modernste Richtung der Pädiatrie, den bakteriologisch-immunologischen Zweig. Mit seinen richtungweisenden Arbeiten auf diesem Gebiet trug er entscheidend dazu bei, dass Wien zu Beginn des 20. Jahrhunderts ,internationales Zentrum modernster immunbiologischer Forschung" [4] wurde.

Den Ausgangspunkt seiner wissenschaftlichen Studien bildeten verschiedene Beobachtungen von unterschiedlichen Verläufen infektiöser und nicht-infektiöser Erkrankungen am Krankenbett. Diese vertiefte Pirquet durch gezielte klinische Experimente, in deren Rahmen er sich intensiv mit Fragen zur Inkubationszeit, zu Infektionstheorien, zu den Vorgängen bei der Vakzination und Revakzination und besonders $\mathrm{zu}$ den Ursachen unterschiedlicher Verläufe und Ausprägungen von Krankheitserscheinungen beschäftigte. Dies fand seinen Niederschlag in zahlreichen Publikationen, unter anderem ,Zur Theorie der Inkubationszeit" [5, 6], ,Zur Theorie der Vakzination“ [7], ,Zur Frage des Aggressins“ [8], „Über die frühzeitige Reaktion der Schutzpockenimpfung" [9] oder zum Thema „Überempfindlichkeit und beschleunigte Reaktion“ [10]. Gemeinsam mit seinem engen Mitarbeiter und Freund Bela Schick beschrieb Pirquet die „Serumkrankheit“ [11] und erarbeitete die Grundlagen zur Tuberkulinreaktion [12]. Diese Arbeiten lieferten ganz wesentliche Beiträge zur Aufklärung pathophysiologischer Zusammenhänge und können zu den Pionierleistungen der modernen Immunologie gerechnet werden.

Sämtliche Untersuchungen aus dieser Zeit zielten darauf $\mathrm{ab}$, zu einem vertieften Verständnis der beobachtbaren klinisch-pathologischen Krankheitserscheinungen zu kommen. Der Schlüssel dafür lag für Pirquet in der Reaktionsfähigkeit des Organismus, welche er von Beginn an ins Zentrum seiner Betrachtungen rückte. Die Beobachtung, dass Personen beim wiederholten Kontakt mit einem Fremdkörper anders reagieren als solche, die zum ersten Mal damit in Berührung kommen, veranlasste 


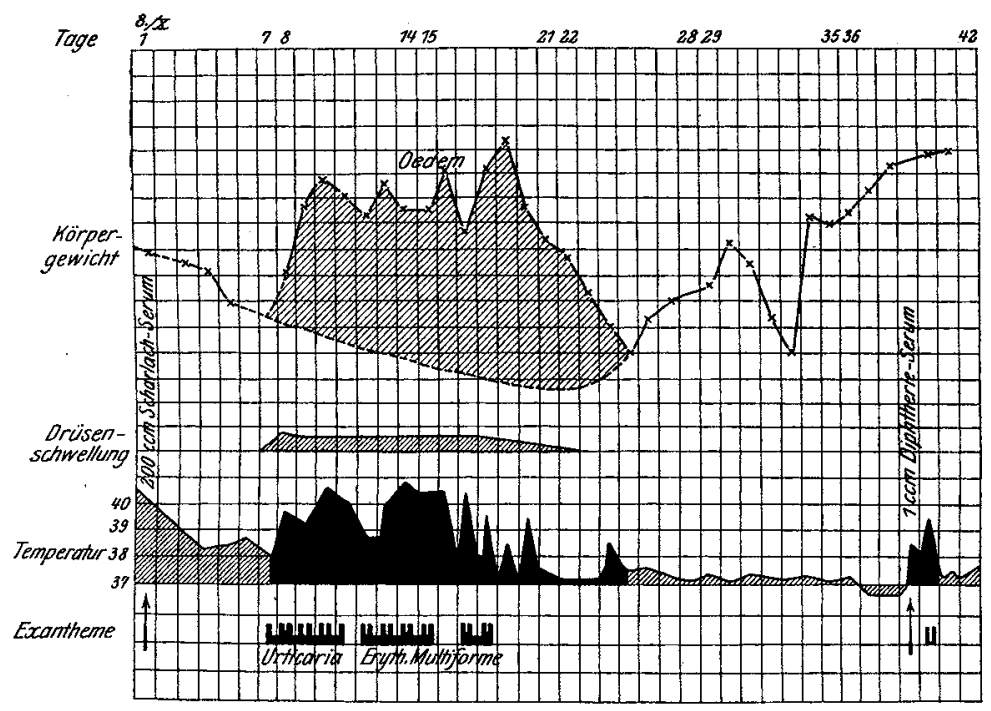

Abb. 1. Serumkrankheit: normalzeitige Reaktion nach erster, sofortige Reaktion nach zweiter Seruminjektion (aus: von Pirquet C [1908] Allergie. Ergeb Inn Med Kinderheilkd 1: 420-464)

Pirquet, von der normalen Reaktion zunächst eine beschleunigte, eine verstärkte und eine Sofortreaktion $\mathrm{zu}$ unterscheiden, bevor er dann im Jahr 1906 den Begriff „Allergie“ schuf, um damit ganz allgemein die zeitlich, quantitativ und/oder qualitativ veränderte Reaktionsfähigkeit des Organismus zu bezeichnen. Die Erkenntnis von der Veränderbarkeit der Reaktionsfähigkeit ist das hervortretende Motiv, das sämtliche Arbeiten durchzieht, die der Einführung des Allergiebegriffs vorausgingen. Ihr liegt der zentrale Gedanke zugrunde, dass dem Organismus selbst eine entscheidende Rolle im Krankheitsgeschehen zukommt. Pirquet mag nicht der erste gewesen sein, der auf die Bedeutung des menschlichen Organismus bei der Entstehung von (Infektions-) Krankheiten hingewiesen hat. Es muss jedoch als seine große Leistung gewürdigt werden, gerade in einer Zeit die Aufmerksamkeit auf den Organismus und seine Reaktionsfähigkeit gerichtet zu haben, in der man aufgrund großer Fortschritte in der bakteriologischen und infektiologischen Forschung vielfach die Ansicht vertrat, dass die gefundenen mikrobiellen Krankheitserreger und auch andere Fremdsubstanzen (z.B. Toxine, Serum etc.) alleine für die durch sie hervorgerufenen Krankheitserscheinungen verantwortlich seien. Pirquet stellte sich mit seiner Auffassung gegen diese einseitige Haltung und konnte damit zu einer ganz entscheidenden Veränderung der Perspektive beitragen. Sie rückte zum einen die Pathogenese der Infektionskrankheiten in ein anderes Licht und gab ihrer Pathologie dadurch eine neue Grundlage, zum anderen konnte er auf diese Weise die medizinische Forschung auch auf vielen anderen Gebieten nachhaltig befruchten. Mit der Orientierung auf den Organismus kristallisiert sich bereits in einer seiner frühesten Arbeiten aus dem Jahr 1903 [5, 6] ein wesentlicher Grundgedanke heraus, der dem gesamten, im Weiteren entwickelten Allergiekonzept zugrunde liegt und sich bis in die letzten Arbeiten über die Allergie des Lebensalters [13, 14] hinein fortsetzt.

\section{Der Allergiebegriff 1906}

Mit einem kurzen Artikel in der Münchner medizinischen Wochenschrift vom 24. Juli 1906 [15] führte Pirquet den Begriff „Allergie“ offiziell in die medizinische Fachwelt ein. Das erste Mal allerdings, dass dieser damals völlig neuartige Ausdruck überhaupt in einer wissenschaftlichen Publikation auftauchte, war bereits zwölf Tage früher in einem Artikel in der Wiener klinischen Wochenschrift [9], wo Pirquet den Leser in einer unauffälligen Fußnote auf seine demnächst erscheinende Habilitationsschrift mit dem Titel „Klinische Studien über Vakzination und vakzinale Allergie" [16] hinwies, ohne auf den bis dahin unbekannten Begriff näher einzugehen.

Der Grund für die neue Wortschöpfung war Pirquets Bedürfnis nach einem allgemeinen Ausdruck zur Beschreibung des Phänomens der veränderten Reaktionsfähigkeit des Organismus. Alle bisherigen Termini schienen ihm nur eine einseitige Bedeutung zu haben, er dagegen hielt es für das Wichtigste, einmal ohne vorgefasste Meinung die klinische Änderung der Reaktionsfähigkeit zu studieren. Der Begriff Allergie war damit zunächst ein rein klinischer Ausdruck „ohne jedes bakteriologische, pathologische oder biologische Vorurteil“" [17]. Pirquet wies in seinen Ausführungen von 1908 darauf hin, dass der zugrunde liegende Mechanismus von Erscheinungen wie Immunität oder verstärkter Empfindlichkeit die medizinische Wissenschaft bereits seit einem halben Jahrhundert beschäftigen würde. „Die großen Entdeckungen welche diesbezüglich in der Erkenntnis der pathogenen Bakterien, sowie der Formelemente und der löslichen Stoffe des Blutes gemacht worden sind, haben aber die Aufmerksamkeit hauptsächlich auf die mikroskopische und tierexperimentelle Beobachtungsweise gelenkt, während die klinische verhältnismäßig vernachlässigt wurde." [17] Aus letzterer heraus entwickelte Pirquet seinen neuen Begriff.

Die ursprüngliche Einführung des Allergiebegriffs sei hier im originalen Wortlaut wiedergegeben: „(...) Wir 
Aus der k. k. Universitäts-Kinderklinik in Wien (Vorst. : Hofrat Escherich).

Allergie.

Von C. v. Pirquet.

In den letzten Jahren ist eine Reihe von Tatsachen gesammelt worden, welche in das Bereich der Immunitätslehre gehören, aber unter diesen Namen schlecht passen: die Befunde von Ueberempfindlichkeit am immunisierten Organismus. ${ }^{1)}$

Diese beiden Ausdrücke schreien gegeneinander; unter immun stellen wir uns doch einen Organismus vor, welcher gegen eine Krankheit geschützt ist, von ihr nicht mehr angegriffen wird; und der soll gleichzeitig gegenüber derselben Krankheit überempfindlich sein?

Diesen Widerspruch hat schon v. Be h ring gefühlt, als er den Tod von gegen Tetanus hoch immunisierten Tieren an kleinen Mengen desselben Toxins als "paradoxe Reaktion" bezeichnete.

Eine "Paradoxie“ können wir doch nur als Ausnahmefall gelten lassen; je mehr man aber in dieses Gebiet eindringt, desto weiter reicht die Gesetzmässigkeit, und wir kennen schon jetzt eine grosse Zahl von Krankheitsprozessen, bei denen Symptome von Ueberempfindlichkeit angetroffen werden. Hieher gehören:

Tetanus (v. Behring, K r etz). Tuberkulose (Cour mont, Strauss und Gamaleia, Babes und Proca, Detre-Deutsch, B. Schick, Löwenstein und Rap paport, Mölier, Löwenste in und Ostrows ky), Syphilis (Finger und Land ste in er), Diphtherie (Rigt), Serum (Arthus, v. Pirquet und Schick, Lehndorff, B. Otto, Rosena und Anderson), Bakterien im allgemeinen, Organextrakte, diverse Eiweissubstanzen, Heufieber (A. W o $1 \mathrm{f} \mathrm{f}$ - E i s n e r).

Sind aber wirklich Immunität und Ueberempfindlichkeit mit einander verbunden, oder sind die Prozesse, bei denen Vorbehandlung Immunität verursacht, von jenen abzutrennen, wo sie zur Ueberempfindlichkeit führt?

A. W o If $\mathrm{f}-\mathrm{E}$ is $n \mathrm{e}^{2}$ ) will diese Trennung durchführen: die Prozesse, bei denen Toxine beteiligt sind, führen zu Antitoxinbildung und Immunität, die Prozesse, in welchen Endotoxine das wirksame Agens vorstellen, führen zur Ueberempfindlichkeit.

Wir sehen aber schon aus den Erfahrungen beim Tetanus, dass bei rein antitoxischen Prozessen Ueberempfindlichkeit

1) Vergl. v. Pirquet und $S \mathrm{chick}$ : Ueberempfindlichkeit und beschleunigte Reaktion. Münch. med. Wochenschr. 1906, 2.

$\left.{ }^{2}\right)$ Zentralbl. f. Bakteriolog. Bd. 37, 1904; Münch. med. Wochenschrift 1906, No. 5; Das Heufieber, München, Lehmann 1906.

Abb. 2. „Allergie“ (aus: von Pirquet C [1906] Allergie. Münch Med Wochenschr 53: 1457-1458)

brauchen ein neues, allgemeines, nicht präjudizierendes Wort für die Zustandsänderung, die der Organismus durch die Bekanntschaft mit irgend einem organischen, lebenden oder leblosen Gifte erfährt. Der Geimpfte verhält sich gegenüber der Lymphe, der Luetische gegenüber dem Syphilisvirus, der Tuberkulöse gegenüber dem Tuberkulin, der mit Serum Injizierte gegenüber dem Serum anders als ein Individuum, welches mit dem betreffenden Agens noch nicht in Berührung gekommen ist; er ist deswegen noch weit entfernt, unempfindlich zu sein. Alles was wir von ihm sagen können, ist, dass seine Reaktionsfähigkeit geändert ist. Für diesen allgemeinen Begriff der veränderten Reaktionsfähigkeit schlage ich den Ausdruck Allergie vor. Allos bezeichnet die Abweichung von der ursprünglichen Verfassung, von dem Verhalten des Normalen, wie in Allorhythmie, Allotropie. Der Geimpfte, der Tuberkulöse, der mit Serum Injizierte werden den respektiven Fremdkörpern gegenüber allergisch. (...)“ [15]
Pirquet kreierte den Ausdruck „Allergie“ aus den griechischen Wörtern ,allos“ und „,ergon“. „Allos“ bedeutet ,anders“ und ,ergon“ steht unter anderem für „Tätigkeit, Verrichtung, Handlung“, d.h. für aktive Vorgänge $[18,19]$. ,Mit dem Wort Ergeia ist der Begriff der aktiven Reaktionsfähigkeit betont." [20] Aufgrund seiner klinischen Beobachtungen und theoretischen Überlegungen war für Pirquet die Tatsache entscheidend, dass sich der Organismus selbst aktiv am Krankheitsgeschehen beteiligt, dass er selbst wirksam ist und durch seine Tätigkeit (Reaktion auf Krankheitsauslöser) das klinisch-pathologische Erscheinungsbild mitbestimmt. Der Begriff „Allergie" bringt die veränderte Reaktionsfähigkeit zum Ausdruck, ,die Abweichung von der ursprünglichen Verfassung, von dem Verhalten des Normalen" [15]. Dabei verstand Pirquet unter ,normal“ den Zustand eines Organismus, der mit der betreffenden Substanz noch nicht in Berührung gekommen ist, wie beispielsweise der Erstvakzinierte mit dem Impfstoff, und unterschied in diesem Sinn eine normale (,normergische“ [21]) von einer allergischen Reaktion.

Pirquet unterteilte die allergischen Reaktionen nach verschiedenen Gesichtspunkten. Entsprechend seiner Definition, die er in dieser Form erstmals 1908 gab [17], umfasst Allergie folgende Teilphänomene:

\section{Zeitlich veränderte Reaktionsfähigkeit:}

1. Frühreaktion (sofortige Reaktion innerhalb 24 Stunden)

2. Torpide Frühreaktion (am 2.-4. Tag)

3. Beschleunigte Reaktion (am 4.-6. Tag)

II. Quantitativ veränderte Reaktionsfähigkeit:

1. Verstärkte Reaktionsfähigkeit (Überempfindlichkeit, paradoxe Reaktion, Anaphylaxie)

2. Abgeschwächte Reaktionsfähigkeit (Unterempfindlichkeit)

3. Aufgehobene Reaktionsfähigkeit (Unempfindlichkeit, Immunität)

III. Qualitativ veränderte Reaktionsfähigkeit:

Erkennbar an der veränderten klinischen Erscheinung (Manifestation) der Reaktion

Diese Aufstellung Pirquets macht deutlich, dass er den Allergiebegriff ganz allgemein dachte und als übergeordneten Begriff verstand, der grundsätzlich sämtliche Änderungen der Reaktionsfähigkeit mit einschließt. Ausdrücke wie „Überempfindlichkeit“ oder „Anaphylaxie“ sind mit dem der Allergie nicht identisch. Es handelt sich dabei nach Pirquets eigenen Worten um „Einzelfälle der Allergie“, welche auf einer besonderen Veränderung der Reaktionsfähigkeit in eine bestimmte „Richtung“ beruhen [20]. Eine Einschränkung des Allergiebegriffs auf eine bestimmte Richtung, wie es bei seiner heutigen Verwendung der Fall ist, wird dem Allergiebegriff im ursprünglichen (Pirquet'schen) Sinn daher nicht gerecht. Zu bemerken ist an dieser Stelle auch, dass sich Pirquet damit eindeutig aussprach, wie andere Begriffe (z.B. Anaphylaxie) zu seinem Allergiebegriff stehen. Was er auf diese Weise eigentlich geklärt hatte, wurde von vielen nach ihm nicht übernommen, weshalb das Problem der begrifflichen Verwirrung in der „allergischen“ Nomenklatur bestehen blieb/bleibt. 


\section{Mechanismus der Allergie}

Obwohl Pirquet von der klinischen Betrachtung ausging, war es ihm selbst sehr daran gelegen, die der Allergie zugrunde liegenden Mechanismen aufzuklären. Er beschränkte sich dabei auf seine klinischen Experimente u.a. mit der Serumtherapie, der Vakzination und der Tuberkulinreaktion, bezog zum Verständnis und zur Erklärung seiner Beobachtungen aber auch die aus der mikrobiologischen, immunologischen und tierexperimentellen Forschung stammenden Ergebnisse mit ein. „(...) Der Standpunkt der Allergie bewirkte, dass wir unser Hauptaugenmerk auf die klinischen Veränderungen der Reaktion legten ohne Rücksicht, ob sich darin ein Schutz des Organismus aussprach, oder ob andere Verhältnisse zutage treten. Zum Teile betrachteten wir alte Tatsachen in neuem Licht, zum anderen Teile aber kamen eben durch diese Forschungsweise neue Phänomene zutage, die wir hauptsächlich nach klinischer Richtung zu analysieren trachteten. Das Schema, das wir an alle Krankheiten anlegten, war die theoretische Einteilung des Allergiebegriffs, der veränderten Reaktionsfähigkeit nach Zeit, Quantität und Qualität.“ [22]

Mit dem Begriff „Allergie“ beschrieb Pirquet „die Zustandsänderung, die der Organismus durch die Bekanntschaft mit irgend einem (...) Gifte erfährt" [15] und brachte damit sprachlich zum Ausdruck, was er aus seinen klinischen Beobachtungen folgerte, nämlich eine Reaktionsänderung als Folge einer „Bekanntschaft“ des Organismus mit einem Fremdkörper, z.B. durch Vorbehandlung, vorausgehende Infektion mit demselben Erreger oder Vakzination. Die Problematik dieser und anderer Aussagen Pirquets zu diesem entscheidenden Punkt der Allergielehre besteht darin, dass aus ihnen nicht eindeutig hervor geht, von wem oder was die Änderung der Reaktionsfähigkeit bewirkt wird. Sie bergen damit in sich die Gefahr von Missverständnissen. Wenn der Organismus eine Zustandsänderung „erfährt“, wenn seine Reaktionsfähigkeit „,geändert/verändert ist", dann könnte das auch in dem Sinn aufgefasst werden, dass an dem ganzen Prozess der Reaktionsfähigkeitsänderung der Organismus selbst nur passiv beteiligt ist. Letztlich muss aber festgestellt werden - und diesbezüglich liefert die moderne Immunologie die nötige Erkenntnisgrundlage -, dass der Organismus tatsächlich selbst aktiv für die Reaktionsänderung verantwortlich ist. Der Kontakt mit dem Fremdkörper stellt den Anlass (,Auslöser“") dafür dar, im Rahmen der Auseinandersetzung mit demselben ändert der Organismus seine Reaktionsfähigkeit.

Man darf wohl davon ausgehen, dass sich Pirquet selbst im Klaren war über die Tatsache, dass der Organismus die Änderung seiner Reaktionsfähigkeit aktiv vollzieht. Einerseits sprach er von einer ,aktiven Reaktionsfähigkeit“" [20] des Organismus, zum anderen sah er die Allergie im Zusammenhang mit der „Antikörperbildung“ [17] - und diese ist zweifelsfrei eine aktive Leistung desselben. Hinzu kommt, dass die Gesamtauffassung, die Pirquet in seinen Arbeiten über das Allergiethema vertrat, von der Orientierung auf den Organismus und dessen aktive Rolle im Krankheitsgeschehen geprägt ist. Dass Pirquet dies nicht ausdrücklicher formulierte, mag an seiner Betrachtungsweise liegen. Durch seine klinischen Be- obachtungen nahm er im Prinzip lediglich das Ergebnis der Reaktionsfähigkeitsänderung wahr und nicht den Prozess der Veränderung direkt. Dies könnte seine Ausdrucks- und Darstellungsweise erklären. - Die in diesem Zusammenhang zwangsläufig auftretende und für das Gesamtverständnis letztlich unumgängliche Frage nach dem Wesen des Organismus geht weit über die Allergielehre Pirquets hinaus und kann hier darum nur angesprochen werden.

Den Ausführungen dieser frühen Jahre ist zu entnehmen, dass für Pirquet die Änderung der Reaktionsfähigkeit vom Kontakt mit einem äußeren Faktor abhing. Das muss betont werden, da die im Jahr 1929 vorgenommene Erweiterung des Allergiebegriffs gerade diesen Punkt betrifft (s.u.). Die äußeren Faktoren (Fremdkörper), welche den Organismus durch ein- oder mehrmalige Einverleibung zu einer Veränderung der Reaktivität veranlassen, bezeichnete Pirquet als „Allergene“. ,Der Ausdruck ist (...) an die Bezeichnung Antigen angelehnt, welcher eine Substanz bedeutet, die Antikörper zu erzeugen vermag. Der Begriff Allergen reicht weiter: Außer den Antigenen gehören zu den Allergenen die zahlreichen Eiweißkörper welche keine Antikörperbildung, aber Überempfindlichkeit verursachen. Allergene sind alle Erreger von Infektionskrankheiten, die von Immunität gefolgt sind; zu den Allergenen werden auch die Gifte von Mücken und Bienen zu rechnen sein, insoweit hiernach Erscheinungen von Unter- oder Überempfindlichkeit auftreten. Aus dem Grund werden wir auch die Pollen des Heufiebers, die Urtikaria erzeugenden Substanzen der Erdbeeren und Krebse, wahrscheinlich auch eine Reihe organischer Substanzen, welche zu Idiosynkrasien führen, unter diesem Namen vereinigen können." [15] Wie der Allergiebegriff war demnach auch der Ausdruck „Allergen“ ein ganz umfassender, der von harmlosen Umweltsubstanzen bis zu Infektionserregern reichte.

Nach der Auffassung Pirquets über die Entstehung der Krankheitserscheinungen wirkte der Krankheitserreger/-auslöser (,Allergen“ nach Pirquet) nicht unmittelbar auf den Organismus, sondern musste erst verändert werden, um wirksam zu sein. „Die Veränderung des Allergens erfolgt durch spezifische, antikörperartige Reaktionsprodukte, die der Organismus auf den Reiz des Allergens hin bildet." [17] Pirquet ließ es dahingestellt, wie man sich diese Veränderung vorzustellen habe und betonte hauptsächlich, dass der Zeitpunkt, wo Antikörper und Antigen zusammentreten, auch der Zeitpunkt sei, an dem sich die klinischen Krankheitserscheinungen manifestieren würden. Dass die Erscheinungen durch antikörperartige Reaktionsprodukte bedingt sein könnten, hatten Pirquet und Schick hauptsächlich aus den zeitlichen Analogien zwischen Antikörperbildung und der Ausbildung der Allergie geschlossen.

Entscheidend ist, dass der von Pirquet häufig gebrauchte Begriff „Antikörper“ nicht mit der heutigen, eindeutig definierten Bedeutung (Immunglobulin) gleich gesetzt werden darf. Und auch das, was er unter AntigenAntikörper-Reaktion verstand, ist nicht mit der entsprechenden modernen Vorstellung identisch bzw. darauf beschränkt. Pirquet wies selbst wiederholt darauf hin, dass die echte Natur der „Antikörper“ noch nicht geklärt sei und verwendete deshalb stellvertretend regelmäßig auch 
verschiedene andere, weniger definierte Ausdrücke (z.B. „Reaktionsprodukte des betroffenen Organismus" [5]). Teilweise bezeichnete er sie auch als „Ergine“ [17], wobei sich dieser Begriff weder durchgesetzt noch lange Zeit gehalten hat. Das, was Pirquet damit allerdings ausdrücken wollte, ist auch aus heutiger Sicht noch gültig. Für ihn war das Ergin ,die Substanz, welche im Organismus Trägerin der Allergie ist“. „Ich verstehe unter Ergin diejenige hypothetische Substanz, welche die klinische Wirkung des Allergens auf den Organismus vermittelt." Und weiter: „Ich verstehe darunter Körper, welche bei der klinischen Reaktionsfähigkeit des Organismus intervenieren, ohne dass ich vor der Hand einen bestimmten chemischen oder biologischen Charakter an den Namen knüpfe.“ [17] Diese Aussagen können die grundsätzliche Haltung Pirquets verdeutlichen und zeigen, dass seine Vorstellungen über den Mechanismus der Allergie durchwegs einen hypothetischen Charakter haben. Er brachte offen zum Ausdruck, dass es fraglich sei, ob man die verschiedenen Anzeichen der Allergie auf ,einheitliche Körper“ zurückführen könne. „Ich glaube nämlich, dass die genaue Erforschung der Allergie bei den verschiedenen Prozessen sehr komplizierte und ungleichartige Verhältnisse aufdecken wird.“ [17] Damit wies Pirquet weit in die Zukunft und vermied zu einem Zeitpunkt, an dem die immunologischen Vorgänge noch weitestgehend unbekannt waren, explizit eine zu festgefahrene Definition oder Erklärung des zugrunde liegenden Mechanismus. Seinem Standpunkt als klinischer Forscher wurde diese Haltung absolut gerecht. Aus Sicht der modernen Immunologie ist mittlerweile natürlich geklärt, dass nicht alle von ihm beschriebenen Prozesse auch tatsächlich durch Antikörper (Immunglobuline) vermittelt werden. So liegt beispielsweise der Tuberkulinreaktion eine zelluläre Immunreaktion zugrunde, wobei die „Wirkung“ der beteiligten Zellen ohne weiteres der Pirquet'schen Vorstellung von „Antikörpern“ entspricht. Der Antikörperbegriff Pirquets umfasst demnach neben den heutigen Immunglobulinen auch die Zellen des Immunsystems (insb. T-Lymphozyten) und ist damit weder auf das humorale noch auf das zelluläre Teilsystem beschränkt.

\section{Diagnostische Verwertung der Allergie}

Noch mehr als an der Aufklärung der zugrunde liegenden Mechanismen war Pirquet als Kliniker an der praktischen Verwertung der Kenntnisse allergischer Vorgänge interessiert. Die charakteristischen Erscheinungen, welche ein Organismus, der eine Krankheit bereits durchgemacht hat, nach neuerlichem Einverleiben der betreffenden Krankheitserreger darbietet, bezeichnete er als „Allergiereaktionen“ [23]. Ganz allgemein dienten sie ihm zur Diagnose eines vorausgegangenen Allergenkontaktes.

Ein Paradebeispiel der diagnostischen Allergiereaktionen ist die kutane Tuberkulinreaktion, deren Methode Pirquet von den Erfahrungen bei der Kuhpockenimpfung ableitete. So wie das Auftreten einer frühzeitigen, papulösen Reaktion nach dieser Impfung beweist, dass der Organismus den Vakzinationsprozess schon einmal durchgemacht hat, beantwortet der tuberkulöse (d.h. der mit Tuberkuloseerregern schon einmal infizierte) Organismus in analoger Weise die kutane Impfung mit Tuberkulin durch rasche Bildung einer Papel an der Impfstelle. „Ein Organismus, welcher noch nicht mit Tuberkulose in Berührung gekommen ist, ist gegen Tuberkulin unempfindlich, er erwirbt die Empfindlichkeit oder Reaktionsfähigkeit nur dadurch, dass er eine tuberkulöse Erkrankung durchmacht. In der Natur geschieht dies nur infolge der Infektion mit Tuberkelbazillen, artifiziell kann die Reaktionsfähigkeit auch durch Injektion abgetöteter Tuberkelbazillen oder ähnliche Maßnahmen erzeugt werden." [24] Die Kutanreaktion ermöglichte es auf einfache Weise, das biologische Verhalten des Organismus gegen Tuberkuloseerreger festzustellen. Eine positive Tuberkulinreaktion weist auf das Vorhandensein von tuberkulösen Veränderungen bzw. auf eine früher durchgemachte Tuberkulose hin, während eine negative Tuberkulinreaktion einen bestehenden oder früheren Kontakt mit den Tuberkuloseerregern im Allgemeinen ausschließt. Mayerhofer, selbst Schüler und Mitarbeiter Pirquets, bezeichnete später die Tuberkulinprobe ganz im Sinne desselben als ,eine biologische Frage an den Organismus, die biologisch beantwortet wird und nur biologisch verstanden werden kann“" [25].

Aufgrund der Häufigkeit der Tuberkulose in der damaligen Zeit und der einfachen Handhabbarkeit dieses Untersuchungsverfahrens erfuhr die Tuberkulinprobe nach ihrer Einführung 1907 eine sehr weite Verbreitung und verhalf Pirquet zu weltweiter Popularität. Im Zusammenhang mit dem Allergiebegriff ist die Tuberkulinreaktion deswegen interessant, weil sie diesen an einem wohlbekannten Phänomen eindrucksvoll illustriert, wozu auch ihre charakteristischen Verläufe unter verschiedenen Bedingungen beitragen. Bei Untersuchungen mit der kutanen Tuberkulinreaktion stellte Pirquet fest, dass diese unter bestimmten Umständen trotz bestehender Tuberkulose negativ ausfallen kann. Sie bot Pirquet die einfache Möglichkeit, durch tägliche Auslösung derselben die Reaktionsfähigkeit des Organismus kontinuierlich zu messen und so ein zusammenhängendes Bild des Verlaufs der „tuberkulösen Allergie“ zu erhalten [26]. Auf diese Weise konnte er zeigen, dass die Reaktion bei kachektischen Individuen im letzten Stadium der Tuberkulose und ,sehr häufig in den letzten zehn Lebenstagen von Kindern mit Miliartuberkulose und Meningitis tuberculosa" erlischt [22]. Daneben beobachtete Pirquet das komplette Verschwinden der Reaktionsfähigkeit auch im Rahmen der Maserninfektion, was er eingehend studierte [27, 28].

Das charakteristische Verhalten der kutanen Tuberkulinreaktion während der Masern, das auch in späteren, systematisch angelegten Studien immer wieder bestätigt werden konnte (z.B. [29]), ist durch eine deutliche Abschwächung der Reaktion in den Tagen vor Ausbruch des Masernexanthems, eine völlige Reaktionslosigkeit während der Exanthemphase und ein Zunehmen der Reaktionsfähigkeit nach Überstehen der Masern gekennzeichnet. Die Zeit der ,mangelhaften Reaktionsfähigkeit des Organismus (Anergie)“ bezeichnete Pirquet als ,anergische Periode" [28] und brachte die nachweisbare Reaktionsverminderung im Verlauf der Maserninfektion mit der Erfahrungstatsache zusammen, dass der tuberkulöse Prozess während der Masern sehr häufig an Ausbreitung gewinnt. Zudem machte er die Reaktionslosigkeit bzw. 


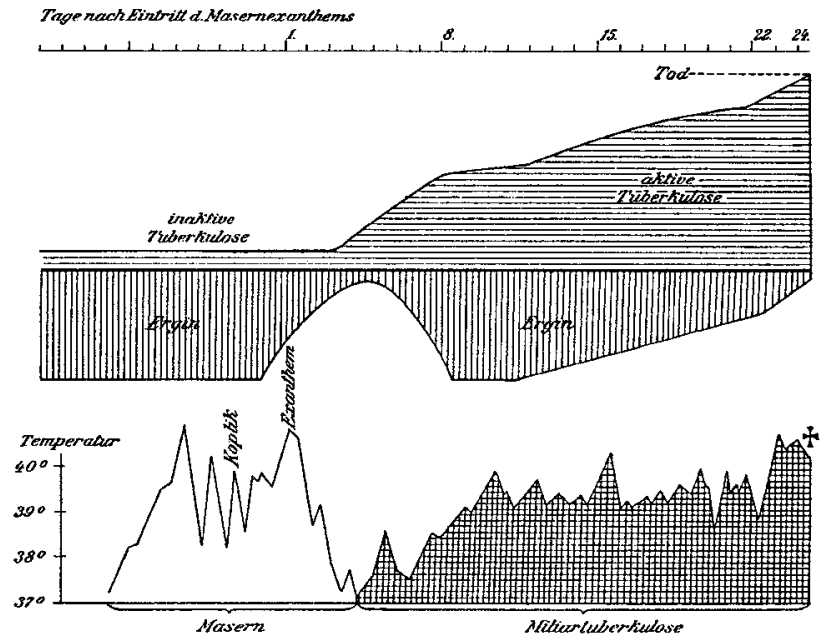

Abb. 3. Schematische Darstellung der Einwirkung von Masern auf Tuberkulose (aus: von Pirquet C [1911] Masern, 2. Aufl. A. Hölder, Wien. In: Nothnagel H (Hrsg), Spezielle Pathologie und Therapie. IV. Band, II. Teil. A. Hölder, Wien, 1913, S. 1-196)

die verminderte Reaktionsfähigkeit dafür verantwortlich, dass der Organismus während der anergischen Periode ,den verschiedensten Infektionen ziemlich schutzlos preisgegeben ist“" [28], welche dann zu den sekundären Komplikationen der Masernerkrankung führen könnten.

Pirquet versuchte die Beobachtungen aus seinem Verständnis der allergischen Prozesse heraus zu erklären. Er spekulierte, dass die Abnahme der tuberkulösen Allergie während der Masern ,,durch eine Absorption der Ergine“, „durch das Fehlen eines anderen zur Reaktion notwendigen chemischen Bestandteils oder durch die Anwesenheit eines hindernden Elements bedingt sein" könnte [27, 28]. Erst über 80 Jahre später konnte durch molekularbiologische Untersuchungen der tatsächlich zugrunde liegende Mechanismus aufgeklärt und Pirquets theoretische Überlegungen prinzipiell bestätigt werden. Er beruht auf einer Interaktion zwischen dem Masernvirus und dem Oberflächenmolekül CD46 auf Monozyten/Makrophagen, die über eine Verminderung der IL-12-Produktion in diesen Zellen zu einer Beeinträchtigung zellvermittelter Immunreaktionen führt. Diese Ergebnisse wurden 1996 in der Zeitschrift Science publiziert [30].

\section{Erweiterung des Allergiebegriffs 1929}

Die bis hierher vorgebrachten Ausführungen zur Erläuterung des Pirquet'schen Allergiebegriffs entsprechen der Auffassung, die Pirquet in einem Aufsatz über die Geschichte der Allergie aus dem Jahr 1927 [31] vertrat. Darin fasste er rückblickend die Entstehung der Allergielehre - und dabei vor allem seine eigenen Arbeiten zu diesem Thema - zusammen und schloss mit den Worten: „Wenn ich jetzt, nach vielen Jahren, diese Arbeiten überblicke, so freut es mich, zu konstatieren, dass sie in den damals von mir als sichere Ergebnisse bezeichneten Punkten keine Fehlschlüsse enthalten haben. Der Namen ,Serumkrankheit' und der Begriff der ,Allergie' sind allgemein angenommen worden. Die große Literatur, die seither um diese Fragen entstanden ist, hat meine damaligen Annahmen und Beobachtungen nicht umgestoßen, und das praktisch wichtigste Resultat, die kutane Tuberkulinreaktion, wird von den Kinderärzten der ganzen Welt in demselben Sinne verwendet, wie ich sie damals angegeben hatte." [31] Die Bedeutung dieser Arbeit ist vor allem darin zu sehen, dass sie Pirquets eigenes Verständnis des Allergiebegriffs zu einem Zeitpunkt (1927) wiedergibt, zu dem er selbst das engere immunologische Forschungsfeld, dem die Allergielehre entsprungen war, schon seit vielen Jahren verlassen hatte.

Wie es im ersten Teil über „Leben und Werk“ ausgeführt wurde, war Pirquet in seinen letzten Lebensjahren mit der Bearbeitung statistischer Zusammenhänge beschäftigt. - Es muss an dieser Stelle darauf hingewiesen werden, dass diese späten Forschungen und die daraus hervorgegangenen Veröffentlichungen in ihrer Bedeutung bisher größtenteils verkannt worden sind. In der gesamten Literatur über Pirquet, die für diese Publikation berücksichtigt wurde - besonders auch in den zum Teil recht umfangreichen biographischen Arbeiten über den Wiener Kinderarzt [31-33] -, sind die Ergebnisse seiner letzten Schaffensperiode - wenn überhaupt - lediglich sehr kurz zusammengefasst, ohne sie im Gesamtkontext mit seiner Allergielehre zu diskutieren. Eine kritische Aufarbeitung der grundsätzlichen Vorstellungen, die diesem letzten Teil seines wissenschaftlichen Werks zugrunde liegen, findet nicht statt, weshalb ganz wesentliche Inhalte davon unerkannt bleiben. Im Folgenden wird eine Annäherung an das Spätwerk Pirquets unter dem Gesichtspunkt einer erweiterten Allergielehre versucht.

Den Anstoß für seine späten Studien erhielt Pirquet zum einen durch die Feststellung, dass in der von ihm geleiteten Kinderklinik ,viel mehr lungenkranke Mädchen zu sehen waren als Knaben“" [13] und zum anderen durch die klinische „Beobachtung des Verhaltens verschiedener menschlicher Altersstufen gegenüber der Tuberkulose“ [14]. Dass der Organismus in bestimmten Lebensjahren (-phasen) auf die Krankheitserreger ganz verschieden reagiert, weise auf die untergeordnete Rolle hin, die beispielsweise die Tuberkelbakterien als äußerer Faktor im Krankheitsprozess spielen. „Selbst bei den Infektionskrankheiten ist die früher allgemeine Vorstellung, dass der Infektionserreger als Hauptfaktor in Betracht kommt, nicht richtig: der zweite, meist wesentlichere Faktor ist die Disposition des Individuums, und diese ist wieder vor allem eine Disposition des Lebensalters. “ [14] Im Rahmen der Auswertung einer enorm großen Menge statistischer Daten zu verschiedensten Krankheiten konstatierte er auch bei zahlreichen anderen (auch nicht-infektiösen) Erkrankungen vergleichbare Verhältnisse wie bei der Tuberkulose, und diese allgemeine Bestätigung einer Geschlechts- und Altersverteilung von Krankheiten ließ ihn eine „Krankheitsdisposition nach Lebensalter und Geschlecht" [35] postulieren. Dass Pirquet in diesem $\mathrm{Zu}$ sammenhang den Allergiebegriff wieder aufgriff und von einer „Altersallergie“ und einer „Allergie des Lebensalters" sprach [13,14], bleibt vor dem Hintergrund seiner ursprünglichen, auf die Immunreaktionen im engeren Sinne beschränkten Begriffsauffassung zunächst möglicherweise unverständlich. Bei eingehender Analyse kann die Verwendung des Allergiebegriffs in diesem Kontext 
aber durchaus begründet und sogar als konsequent bezeichnet werden.

In den Publikationen Pirquets zu diesem Thema findet man nur zwei Stellen, an denen er auf die Verwendung des Allergiebegriffs in diesem erweiterten Zusammenhang eingeht und die im Sinn einer Begründung aufgefasst werden können. Ansonsten hielt sich Pirquet mit Erklärungen und Interpretationen seiner statistischen Befunde weitgehend zurück. Die wichtigste Aussage findet sich in einem Artikel, der etwa einen Monat vor seinem Tod in der Wiener klinischen Wochenschrift vom 17. Januar 1929 publiziert wurde. Sie stellt - und das soll hier bewusst zurückhaltend formuliert werden - möglicherweise den Schlüssel zu einem echten Verständnis der späten Arbeiten Pirquets und damit zu einem Verständnis der Erweiterung des Allergiebegriffs dar. Pirquet schreibt dort: „Im Jahre 1906 habe ich den Ausdruck Allergie für den allgemeinen Begriff der veränderten Reaktionsfähigkeit vorgeschlagen. Ich hatte damit die Zustandsänderung im Auge, welche ein Individuum durch die Bekanntschaft oder Vorbehandlung mit einem Fremdkörper irgendwelcher Art erfährt, unter stillschweigender Voraussetzung, dass das Individuum sonst unverändert geblieben ist. In den letzten Jahren habe ich mich Studien darüber zugewandt, inwieweit die Reaktionsänderung gegenüber einer Noxe nicht durch Vorbehandlung bedingt ist, sondern von einer im Organismus spontan vorgegangenen Veränderung herstammt." [13] Die zweite Aussage, aus der Einleitung der 1930 herausgegebenen Monographie stammend, kann die Vorstellung Pirquets verdeutlichen und verständlich machen. „Um die Pubertätszeit ändert sich die Reaktionsform des tuberkulös Infizierten auf die in seinem Körper wohnenden Tuberkelbazillen. Diese Veränderung der Reaktionsart bringe ich ebenso unter den Begriff der Allergie wie die Veränderung der Reaktion gegenüber tuberkulösem Gift, das in die Haut oder unter die Haut gebracht wird." [14]

Obwohl Pirquet selbst keine Stellung zur methodischen Vorgehensweise bei seinen späten Forschungen nahm, kann man doch vermuten, dass sie sich prinzipiell

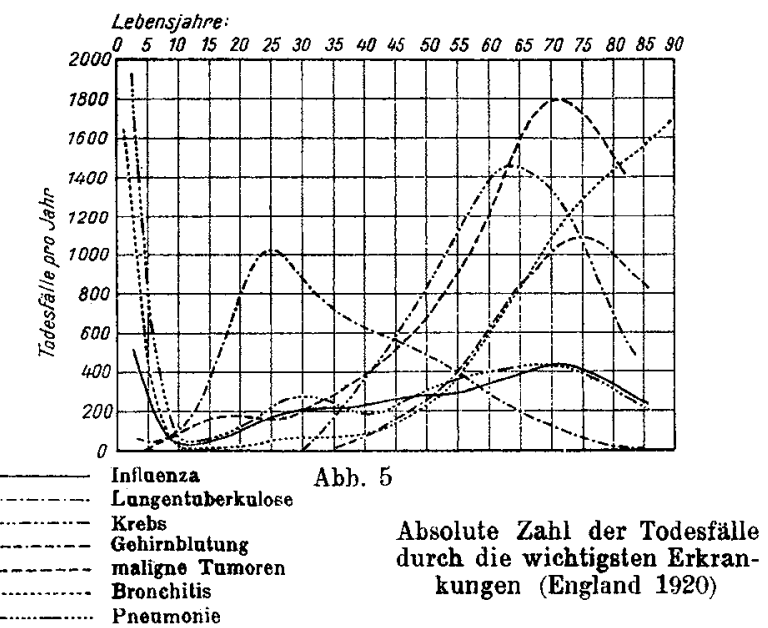

Abb. 4. Altersverteilung verschiedener Krankheiten (aus: von Pirquet C [1929] Allergie des Lebensalters. Wien Klin Wochenschr 42: 65-67) nicht von der unterscheidet, die auch seine früheren klinisch-immunologischen Arbeiten, aus denen der Allergiebegriff ursprünglich hervorging, kennzeichnet: ausgehend von der klinischen Beobachtung folgte auf eine kritische Interpretation der Befunde ein Erklärungsversuch (Theorie), der wiederum an weiteren Beobachtungen geprüft wurde. Auch wenn Pirquet die klinischen Beobachtungen bei seinen letzten, statistisch angelegten Untersuchungen nicht mehr unmittelbar an den Kranken machte, ging er dennoch von ihnen aus. Die indirekte „klinische Beobachtung" anhand der statistischen Daten ermöglichte ihm, eine riesige Fallzahl, ein viel größeres Krankheitsspektrum und einen erheblich größeren Zeitraum zu überschauen, als es durch eigene klinische Studien je möglich gewesen wäre. Er untersuchte (,,beobachtete“) so den klinischen Verlauf verschiedener Krankheiten, der sich ihm durch Manifestationszeitpunkt, Häufigkeitsgipfel, Mortalitätsgipfel etc. darbot. Dabei wies er für sämtliche Krankheiten eine charakteristische Altersverteilung nach und führte sie auf ein unterschiedliches, sich mit dem Alter veränderndes Verhalten des Organismus gegenüber den einzelnen Krankheitsprozessen zurück. Die an seinen früheren Studien entwickelte Vorstellung, dass der Organismus mit seiner Reaktionsfähigkeit eine wesentliche Rolle für die klinischen Erscheinungen und den Verlauf von Krankheiten spielt, muss für Pirquet auch in diesem Zusammenhang ein leitender Gedanke gewesen sein. Die bei seinen statistischen Analysen beobachtete Verhaltensänderung des Organismus mit dem Begriff Allergie zu versehen, kann aus der Argumentation heraus eigentlich als logische Konsequenz betrachtet werden. Der Begriff Allergie (veränderte Reaktionsfähigkeit), den Pirquet aus den Erscheinungen der Serumkrankheit, der Vakzination oder verschiedener Infektionskrankheiten heraus entwickelte, ist letztlich auch in den statistisch beobachteten Erscheinungen der altersabhängigen Krankheitsverläufe enthalten. Darum ist auch der erweiterte Allergiebegriff, weil er von den klinischen Erscheinungen und Verläufen abgeleitet ist, ein klinischer Begriff.

„Worauf beruht nun die Altersallergie? Das ist eine Frage, die wir heute noch gar nicht beantworten können. Im Falle der Tuberkulose mag die Vorstellung berechtigt sein, dass die Disposition der Pubertätsjahre mit den Keimdrüsen in Zusammenhang steht. Es erscheint mir sehr wahrscheinlich, dass die Endokrinologie und die Vererbungslehre berufen sein werden, vieles zu erklären.“ [13] Pirquet war sich offensichtlich durchaus bewusst über die Tatsache, dass sich die Alters- und Geschlechtsabhängigkeit der Krankheiten nicht allein durch Veränderungen der immunologischen Reaktionsfähigkeit erklären lassen. Er brachte den Allergiebegriff mit der Disposition des Organismus zusammen und so erklärt sich auch, dass er in seinen letzten Arbeiten gleichwertig mit den Ausdrücken „Allergie des Lebensalters“ und „Altersallergie“ die Bezeichnungen „Disposition des Lebensalters“ und „Altersdisposition" gebrauchte [13, 14]. Das Unverständnis bzw. die vielen Missverständnisse, die auf der Identifizierung der Allergie eines Organismus mit dessen Disposition beruhen, sind nachvollziehbar, wenn man den Allergiebegriff auf Immunreaktionen beschränkt wissen will. Berücksichtigt man aber andererseits, dass das Immunsystem nicht das einzige reagierende System des mensch- 
lichen Organismus ist - endokrine und neuro-vegetative Reaktionen seien hier als weitere Beispiele genannt - und dass die Interaktionen aller Systeme maßgeblich für die Disposition eines Individuums verantwortlich sind, dann lässt sich die Ausweitung des Allergiebegriffs auch aus heutiger Sicht ohne weiteres rechtfertigen.

Überblickt man die Erweiterung des Allergiebegriffs durch die Arbeiten der letzten Lebenswochen, dann kann man feststellen, dass das Wesentliche weniger in der (umstrittenen) Ausweitung auf nicht-immunologische Gebiete, ja auf die gesamte Disposition des Menschen liegt, sondern vielmehr in dem grundlegenden Krankheitsverständnis Pirquets zu suchen ist, das er sich im Laufe seines Lebens erarbeitet hatte. Das zentrale Element der Erweiterung ist die Erkenntnis, dass die Änderung der Reaktionsfähigkeit des Organismus auch unabhängig von äußeren Faktoren (den sog. Allergenen) erfolgen kann, dass „die Reaktionsänderung gegenüber einer Noxe (...) von einer im Organismus spontan vorgegangenen Veränderung“ herstammen kann. Die Reaktionsfähigkeit und die Allergie, die sich auf die Krankheitsdisposition auswirkende Änderung derselben, werden dementsprechend nicht nur von äußeren, sondern auch von inneren (organismuseigenen) Faktoren bestimmt. Pirquet lieferte dadurch einen weiteren entscheidenden Beitrag zu einem grundsätzlichen Verständnis pathogenetischer Zusammenhänge. Darüber hinaus demonstrierte er mit seinen letzten Arbeiten eindrucksvoll, dass die Reaktionsfähigkeit des Organismus bei einem viel größeren Krankheitsspektrum mit zu berücksichtigen ist, als es aus den frühen, rein immunologisch orientierten Arbeiten zunächst zu erwarten war. Gerade die von Pirquet ausführlich studierten Tumorerkrankungen seien hier stellvertretend genannt [14]. Die Schwierigkeit einer Erklärung der zugrunde liegenden Mechanismen der „Allergie des Lebensalters“ kommentierte Pirquet bescheiden: „Einstweilen sind wir aber noch nicht so weit, sondern sind noch mit dem Sammeln von Tatsachen beschäftigt; Tatsachen, welche geeignet sind, in unerforschte Gebiete der Medizin zu führen." [13] Ihre weitere Aufklärung war ihm allerdings nicht mehr vergönnt.

Pirquets Arbeiten zur Allergie des Lebensalters sind ferner deshalb zu würdigen, weil in ihnen ein ganzer Forschungszweig der modernen Immunologie veranlagt ist, der sich mit den altersabhängigen Veränderungen der immunologischen Reaktionsfähigkeit und ihren Auswirkungen auf die Disposition zu verschiedenen Krankheiten befasst (u.a. [36-38]). Auch diesbezüglich kann Pirquet als Vordenker verstanden werden.

\section{Allergie und Organismus}

Mit der Erweiterung des Allergiebegriffs kehrte Pirquet kurz vor seinem Tode zum Anfang seiner wissenschaftlichen Laufbahn zurück. Die Gedanken und Ideen, die seine frühen Arbeiten prägen, tauchen in denen der letzten Lebenswochen wieder auf. Es ist jedoch ungenügend, diese Rückkehr zum Ursprung allein am Wort „Allergie" festzumachen, wie es von verschiedenen Autoren vorgenommen wird. Wagner [32] schreibt in seiner Pirquet-Biographie: ,With this return to the doctrine of allergy his life and work was completed and the ring was

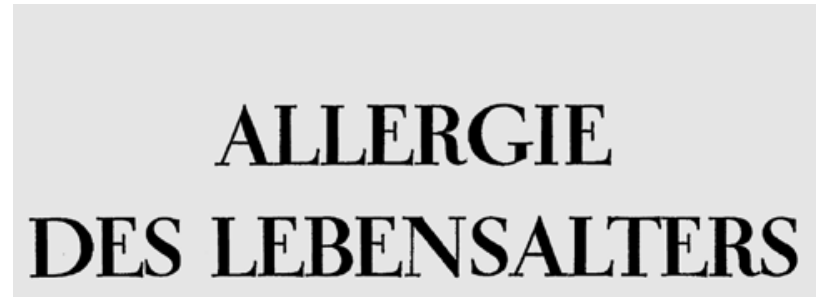

DIE BÖSARTIGEN GESCHWÜLSTE

VON

DR. CLEMENS PIRQUET

O. O. PROTESSOR AN DER UNIVERSITKT WIEN

MIT 142 ABBILDUNGEN UND I TAFEL

I 9

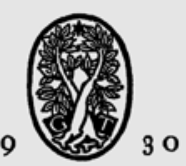

GEORG THIEME / VERLAG / LEIPZIG

Abb. 5. „Allergie des Lebensalters“ (aus: von Pirquet C [1930] Allergie des Lebensalters. Die bösartigen Geschwülste. Thieme, Leipzig)

closed.“ In gleicher Weise äußert sich auch Asperger [1]: „Mit dem Wort ,Allergie“ (aber hier in neuer Bedeutung gebraucht) war Pirquet zu seinen Anfängen zurückgekehrt. Der Kreis war geschlossen.“ Der Allergiebegriff, den Pirquet 1906 einführte und der 1929 wieder auftauchte, ist lediglich der äußerliche Beleg für den ,geschlossenen Kreis“. Erst durch einen tieferen Einblick in die wesentlichen Grundlagen seiner Allergielehre erkennt man mit der zentralen Idee, die das gesamte Werk Pirquets von 1903 bis 1929 durchzieht, den eigentlichen „Kreis“ seiner Forschungen.

Sämtliche Arbeiten, in denen sich Pirquet mit Fragen zur Entstehung und zu den Verläufen von Krankheitserscheinungen auseinandersetzte, zeichnen sich durch eine Hinorientierung auf den Organismus aus. Die Erkenntnis, dass der Organismus am Krankheitsgeschehen selbst aktiv beteiligt ist, stellt den Leitgedanken dar, der die Forschungen des Wiener Kinderarztes prägte. Dies wird nicht nur an den Arbeiten der immunologischen Schaffensperiode (1903-1913) deutlich, sondern das geht auch aus den Veröffentlichungen über die Allergie des Lebensalters (1929) hervor. Gerade darin muss man das Gemeinsame der frühen und der späten Arbeiten zum Allergiebegriff sehen. Dieser Gesichtspunkt soll hier besonders hervorge- 
hoben werden, da er in keiner einzigen der berücksichtigten Quellen, in denen Pirquet und sein Allergiebegriff behandelt werden, in dieser Deutlichkeit ausgesprochen wird. Pirquets Leistung für ein umfassendes Krankheitsverständnis ist die Veränderung der Perspektive. Im Zentrum seiner Allergielehre steht der Organismus, ohne dass damit andere Faktoren (z.B. Fremdkörper oder andere äußere Einflüsse) ausgeschlossen wären oder unberücksichtigt blieben.

Während der Allergiebegriff heute definitionsgemäß auf spezifische Änderungen der Immunitätslage im Sinne einer krankmachenden Überempfindlichkeit und häufig sogar noch strikter allein auf IgE-vermittelte Sofortreaktionen gegen harmlose Umweltantigene beschränkt ist [39, 40], ist die Lehre von der Allergie (,Allergologie“) im Sinne des Pirquet'schen Allergiebegriffs ganz allgemein die Lehre von der Reaktionsfähigkeit des Organismus. Allergie umfasst bei Pirquet als übergeordneter Begriff gleichermaßen Steigerungen und Verminderungen der Reaktionsfähigkeit und damit sowohl „Über-“ als auch „Unterempfindlichkeitsreaktionen“ " [17] und ist darum grundsätzlich geeignet, zu einem gemeinsamen und übergeordneten Verständnis von Erkrankungen verschiedenster Art beizutragen. In diesem weitsichtigen und gleichzeitig herausfordernden Sinne fasste auch Kundratitz [41] die große Bedeutung der von Pirquet in Wien begründeten Allergielehre zusammen: „Pirquets grundlegende Forschungen und deren Ergebnisse (...) wirkten so anregend auf die medizinische Forschung, dass es nun kaum ein zweites Teilgebiet der medizinischen Wissenschaft gibt, das so viele Ärzte und Biologen beschäftigte, das in sämtliche medizinischen Disziplinen und Teilfächer eindrang und das durch seine exakte Bearbeitung bei so vielen Krankheiten bzw. Krankheitserscheinungen in solchem Ausmaße Licht und Klarheit in Bezug auf Ursache, Erscheinungsform, Erscheinungsäußerungen in klinischer, biologischer, pathologisch-physiologischer, pathologisch-anatomischer und serologischer Hinsicht und in Bezug auf Diagnose, Ablauf und Therapie brachte."

\section{Literatur}

1. Asperger H (1974) Clemens von Pirquet zum hundertsten Geburtstag. Padiatr Padol 9 (3): 199-203

2. Huber B (2006) 100 Jahre Allergie: Clemens von Pirquet - Sein Allergiebegriff und das ihm zugrunde liegende Krankheitsverständnis. Teil 1: Leben und Werk. Wien Klin Wochenschr 118: 573-579

3. Silverstein AM (1989) A history of immunology. Academic Press, San Diego

4. Lesky E (1974) Clemens von Pirquet (1874-1929). Öst Ärzteztg 29 (9): 542-545

5. von Pirquet C, Schick B (1903) Zur Theorie der Inkubationszeit (Vorläufige Mitteilung). Wien Klin Wochenschr 16: $758-759$

6. von Pirquet C, Schick B (1903) Zur Theorie der Inkubationszeit. Wien Klin Wochenschr 16: 1244-1247

7. von Pirquet C (1903) Zur Theorie der Vaccination. Wien Klin Wochenschr 16: 1234

8. von Pirquet C, Schick B (1905) Zur Frage des Aggressins. Wien Klin Wochenschr 18: 431-435

9. von Pirquet C (1906) Die frühzeitige Reaktion bei der Schutzpockenimpfung. Wien Klin Wochenschr 19: 855858
10. von Pirquet C, Schick B (1906) Überempfindlichkeit und beschleunigte Reaktion. Münch Med Wochenschr 53: 66-69

11. von Pirquet C, Schick B (1905) Die Serumkrankheit. Franz Deuticke, Wien

12. von Pirquet C (1907) Die kutane Tuberkulinprobe. Med Klin 3 (39): 1197-1199

13. von Pirquet C (1929) Allergie des Lebensalters. Wien Klin Wochenschr 42: 65-67

14. von Pirquet C (1930) Allergie des Lebensalters. Die bösartigen Geschwülste. Thieme, Leipzig

15. von Pirquet C (1906) Allergie. Münch Med Wochenschr 53: $1457-1458$

16. von Pirquet C (1907) Klinische Studien über Vakzination und vakzinale Allergie. Franz Deuticke, Wien

17. von Pirquet C (1908) Allergie. Ergeb Inn Med Kinderheilkd 1: 420-464

18. Pape W (1954) Griechisch-deutsches Handwörterbuch, 3. Aufl. Akademische Druck- und Verlagsanstalt, Graz

19. Gemoll W (1965) Griechisch-deutsches Schul- und Handwörterbuch, 9. Aufl. G. Freytag, München

20. von Pirquet C (1908) Über das Verhalten der menschlichen Haut gegen verschiedene bakterielle Giftstoffe. Wien Klin Wochenschr 21: 915-916

21. von Pirquet C (1906) Ist die vakzinale Frühreaktion spezifisch? Wien Klin Wochenschr 19: 1407-1410

22. von Pirquet C (1910) Allergie. Ergeb Inn Med Kinderheilkd 5: 459-539

23. von Pirquet C (1907) Allergie-Diagnostik. Therap Monatshefte 21: 555-557

24. von Pirquet C (1908) Über das Verhalten der menschlichen Haut gegen bakterielle Giftstoffe. Wien Klin Wochenschr 21: 623-624

25. Mayerhofer E (1929) Pirquet's Allergiebegriff und seine Entwicklung bis 1929. Ergeb Inn Med Kinderheilkd 36: 241-272

26. von Pirquet C (1908) Verlauf der tuberkulösen Allergie bei einem Fall von Masern und Miliartuberkulose. Wien Klin Wochenschr 21: 861-864

27. von Pirquet C (1908) Das Verhalten der kutanen Tuberkulinreaktion während der Masern. Dtsch Med Wochenschr 34: 1297-1300

28. von Pirquet C (1911) Masern, 2. Aufl. A. Hölder, Wien. In: Nothnagel H (Hrsg) Spezielle Pathologie und Therapie. IV. Band, II. Teil. A. Hölder, Wien, 1913 pp 1-196

29. Starr S, Berkovich S (1964) Effects of measles, gammaglobulin-modified measles and vaccine measles on the tuberculin test. N Engl J Med 270: 386-391

30. Karp CL, Wysocka M, Wahl LM, Ahearn JM, Cuomo PJ, Sherry B, et al (1996) Mechanism of suppression of cellmediated immunity by measles virus. Science 273: 228 231

31. von Pirquet C (1927) Zur Geschichte der Allergie. Wien Med Wochenschr 77: 745-748

32. Wagner R (1968) Clemens von Pirquet: his life and work. Johns Hopkins Press, Baltimore

33. Dorffner G, Weippl G (2004) Clemens Freiherr von Pirquet - Ein begnadeter Arzt und genialer Geist. Vier-Viertel-Verlag, Wien

34. Huitema Wemelina (1936) Clemens von Pirquet. Med. Dissertation. van Campen, Amsterdam

35. von Pirquet C (1929) Krankheitsdisposition nach Lebensalter und Geschlecht. Wien Med Wochenschr 79: 18-19

36. Grubeck-Loebenstein B, Wick G (2002) The aging of the immune system. Adv Immunol 80: 243-284 
37. Tarazona R, Solana R, Ouyang Q, Pawelec G (2002) Basic biology and clinical impact of immunosenescence. Exp Gerontol 37: 183-189

38. Burns EA (2004) Effects of aging on immune function. J Nutr Health Aging 8: 9-18

39. Kay AB (2001) Allergy and allergic diseases. First of two parts. N Engl J Med 344: 30-37
40. Abbas AK, Lichtman AH (2003) Cellular and molecular immunology, 5th edn. Saunders, Philadelphia

41. Kundratitz K (1948) Zur Geschichte der Allergie. Wien Klin Wochenschr 60: 794-796

Korrespondenz: Benedikt Huber, Universität Bern, Institut für Medizingeschichte, Bühlstrasse 26, 3012 Bern, Schweiz, E-Mail: benedikt.m.huber@web.de 